\title{
Criteria for the Importance of Multi-Scale Interactions in Turbulent Transport Simulations
}

\author{
A.J. Creely ${ }^{1}$, P. Rodriguez-Fernandez ${ }^{1}$, G.D. Conway ${ }^{2}$, S.J. \\ Freethy $^{1,2}$, N.T. Howard ${ }^{1}$, A.E. White ${ }^{1}$, and the ASDEX \\ Upgrade Team \\ ${ }^{1}$ MIT Plasma Science and Fusion Center, Cambridge, MA 02139, USA. \\ 2 Max Planck Institute for Plasma Physics, Garching 85748, Germany.
}

\begin{abstract}
.
Turbulent transport simulations have been used to develop criteria that indicate when multi-scale turbulent phenomena are important in tokamak plasmas. Eleven experimental plasma discharges from the Alcator C-Mod and ASDEX Upgrade tokamaks are compared to ion- and multi-scale simulations with the Trapped Gyro-Landau Fluid (TGLF) turbulence code. Multi-scale TGLF agrees with all available validation constraints (ion heat flux, electron heat flux, electron temperature fluctuations, and electron perturbative thermal diffusivity) within uncertainty for all cases analyzed. Ion-scale TGLF agrees in only some cases. Two criteria based on the ratios of normalized linear growth rates are able to distinguish cases in which ion-scale simulations are sufficient from cases for which multi-scale simulations are necessary. The form of these criteria reveal the key role of zonal flow mixing in moderating multi-scale effects.
\end{abstract}

PACS numbers: 52.55.Fa, 52.35.Ra, 52.65.Tt, 52.70.Gw

Keywords: Validation, Perturbative Diffusivity, Temperature Fluctuations

Submitted to: Plasma Phys. Control. Fusion 


\section{Introduction}

Understanding the nature of turbulence in fusion plasmas has been one of the central experimental and theoretical challenges of fusion research for many years. Developing accurate models of turbulence and turbulent transport has the dual aims of providing insight into the fundamental nature of turbulent interactions and enabling predictions of the performance of future machines. Before one can in good faith use turbulent transport models to explain turbulent dynamics or predict machine performance, however, one must ensure that these models can correctly reproduce the experimentally measured conditions on existing devices. Validation, the process of determining how accurately a model represents reality insofar as is required for the intended purposes of the model [1], has thus become a key endeavor in fusion energy research.

While it may seem that for the purposes of temperature profile prediction the only relevant parameters for validation are the electron and ion heat fluxes, it turns out that such comparisons are susceptible to fortuitous agreement, and that one must compare many experimentally measured quantities in order to truly assess the accuracy of the model 2, 3. These additional validation constraints include density fluctuations, temperature fluctuations, radial correlation lengths, temperature-density crossphase, the perturbative thermal diffusivity, and others. Generally the more of these constraints that can be applied simultaneously, the more rigorous the validation study.

The highest fidelity turbulent transport models presently applied to fusion plasmas are nonlinear gyrokinetic models, implemented in, for example, the GENE [4] and GYRO [5] codes. These codes can calculate the nonlinear saturated state of plasma turbulence from experimental inputs, but can be quite computationally expensive, requiring hundreds of thousands to tens of millions of CPU hours for a single nonlinear run. Quasi-linear gyrofluid models, on the other hand, use a saturation rule tuned to the results of nonlinear gyrokinetic runs in order to obtain heat fluxes and other turbulence properties from linear growth rates, and require only a few seconds to run [6, 7, 8. The downside of these codes is that one may not trust their outputs outside of the parameter range for which their saturation rule has been tuned.
In addition to choosing a model when investigating turbulence or predicting machine performance, one must also make a number of choices about which physical phenomena to include in a given model. One of the most important of these choices is what range of scales to include in the simulation. For many years, most gyrokinetic simulations were ion-scale, capturing plasma dynamics down to approximately the ion gyroradius. Recent work, however, has shown that in some cases, these ion-scale simulations are unable to accurately reproduce experimental measurements, and that one requires multi-scale simulations, which simultaneously resolve the ion- and electron-scales [9, 10, 11, 12, 13, 14, 15, 16.

Specifically, cases have been identified in which: 1 . Ion-scale simulations miss both heat fluxes and additional constraints such as temperature fluctuations [17] and perturbative diffusivity [18, but multi-scale simulations find good agreement. 2. Ion-scale simulations match heat fluxes, but miss other constraints, while multi-scale simulations match everything [16]. 3. Ionscale simulations simultaneously match heat fluxes and other constraints, indicating that multi-scale effects are unimportant [19]. The intent of this article is to outline a set of criteria that differentiates these cases, and in doing so probe the underlying physical phenomena that govern multi-scale turbulent interactions.

To address this question, this work performed a novel type of validation study, validating the same code on a large number of plasmas from two different machines in order to observe broader trends of when the simulations do and do not agree with experimental measurements. In particular, this work validated TGLF in both ion- and multi-scale configurations in order to investigate when multi-scale effects are important and when ion-scale simulations are sufficient. This validation work utilizes data from eleven discharges on two machines, Alcator C-Mod and ASDEX Upgrade, and applied four validation constraints to each discharge: ion heat flux, electron heat flux, electron temperature fluctuations, and perturbative thermal diffusivity. In addition to developing criteria that describe when multi-scale effects are important, this paper will also validate the most recent incarnation of TGLF, which incorporates the results from multi-scale gyrokinetic simulations [7, in a variety of plasma conditions. Greater detail on the methodology of this validation study is given later in this paper. 
The remainder of this paper is organized as follows. Section 2 describes the two tokamaks used in this study, as well as all of the various diagnostic and analysis techniques used in order to obtain the experimental measurements of the validation constraints. Section 3 then describes the methodology used in this validation study, including a brief description of the eleven plasma discharges and how TGLF was determined to be in or out of agreement with experiment for each plasma condition. Section 4 presents the results of this validation study, as well as the criteria that were developed to determine when multi-scale effects are important. This section also presents profile predictions using ion- and multi-scale TGLF for a few of the discharges. Finally, Section 5 discusses the interpretation and implications of these results.

\section{Machines and Experimental Measurements}

This section describes the experimental hardware (tokamaks and diagnostics) and analysis techniques used to obtain the experimental inputs to and validation constraints for the TGLF simulations.

All experimental measurements in this study were made on Alcator C-Mod and ASDEX Upgrade. Alcator C-Mod [20] is a high field (typical toroidal magnetic field $B_{T}=5.4 \mathrm{~T}$ ), compact (major radius $R=0.67 \mathrm{~m}$ and minor radius $a=0.22 \mathrm{~m}$ ), metalwalled (molybdenum), diverted tokamak. ASDEX Upgrade [21] is a moderate field (typical toroidal magnetic field $B_{T}=2.6 \mathrm{~T}$ ), medium size (major radius $R=1.65 \mathrm{~m}$ and minor radius $a=0.5 \mathrm{~m}$ ), metalwalled (tungsten), diverted tokamak. Note that for typical plasmas on both Alcator C-Mod and ASDEX Upgrade, $\rho_{s} / R \approx 5 \times 10^{-4}$, where $\rho_{s}$ is the ion gyroradius evaluated at the sound speed.

Experimental inputs to TGLF include magnetic equilibria, electron and ion temperature profiles, electron density profiles, plasma rotation profiles, and plasma effective charge $\left(Z_{\text {eff }}\right)$. Each of the measurements described below is accompanied by some level of uncertainty, which is discussed in further detail in Section 3 .

On Alcator C-Mod, the electron temperature profile was measured with a electron cyclotron emission (ECE) grating polychrometer (GPC) 22, 23. Electron density (and also temperature) was measured with Thomson Scattering 24]. Ion temperature and toroidal plasma rotation were measured with an x-ray imaging crystal spectrometer (XICS, also called HiReX) [25]. The effective charge was measured with a visible Bremsstrahlung spectrometer [26]. Magnetic equilibria were reconstructed with EFIT [27.

On ASDEX Upgrade, the electron temperature profile was measured with a heterodyne ECE radiometer 28]. Electron density (and also temperature) was measured with Thomson Scattering [29, 30]. Ion temperature and toroidal plasma rotation were measured with charge exchange recombination spectroscopy (CXRS) 31. The effective charge was measured with CXRS 31 and Bremsstrahlung spectrometers 32. Magnetic equilibria were reconstructed with CLISTE [33].

Heat fluxes are not measured directly, but are calculated using the power-balance solver TRANSP 34. TRANSP takes as inputs all of the profiles that were described above as inputs to TGLF, as well as external heating power and the radiated power profile. Bolometer arrays were used to measure the radiated power profiles on both Alcator C-Mod [35] and ASDEX Upgrade [36]. TRANSP calculates heat fluxes by balancing all power sources (ohmic heating, auxiliary heating, etc.) and sinks (radiated power, convection, electron-ion exchange, etc.) in both the ions and electrons. By propagating the uncertainties in the inputs through the governing equations of TRANSP, one is also able to obtain uncertainties in the experimental heat fluxes.

As has become increasingly clear in recent years, it is important to include more than just heat fluxes in validation studies, in order to avoid fortuitous agreement between the simulation and experiment [2, 3]. For this reason, this study includes electron temperature fluctuations and the electron perturbative thermal diffusivity as validation constraints [17, in addition to electron and ion heat fluxes.

Low-k $\left(k_{\theta} \rho_{s} \lesssim 0.3\right.$, where $k_{\theta}$ is poloidal wavenumber) electron temperature fluctuations, $\widetilde{T} / T$, are measured with correlation electron cyclotron emission (CECE) diagnostics on both Alcator CMod [37, 38, and ASDEX Upgrade [39, 40. CECE diagnostics correlate signals from two closely spaced radiometer channels in order to resolve turbulent temperature fluctuations in the plasma down to a few tenths of a percent while removing thermal noise. The total experimental temperature fluctuation level and its uncertainty, calculated as in Reference [40], are compared to TGLF.

The final validation constraint used in this study is the electron perturbative thermal diffusivity, $\chi_{e}^{\text {pert }}$, which is defined as 41]:

$$
\chi_{e}^{p e r t}=-\frac{1}{n_{e}} \frac{\partial Q_{e}}{\partial \nabla T_{e}}
$$

where $n_{e}$ is the electron density, $Q_{e}$ is the electron heat flux, and $T_{e}$ is the electron density. The perturbative diffusivity therefore measures the extent to which a change in the electron temperature gradient changes the electron heat flux. 
This study uses the propagation of partial sawtooth generated heat pulses in order to measure the perturbative diffusivity [18, 19]. In particular, the Extended-Time-to-Peak calculation method [42] is used on heat pulses generated by partial sawteeth, as these avoid complications associated with full sawteeth 43. The region in which the perturbative diffusivity is measurable is constrained by the sawtooth mixing radius and the radius beyond which the heat pulse is too small to distinguish from noise.

\section{Experimental Plasmas and Validation Methodology}

This section describes the eleven experimental plasma discharges that were used in this study, as well as the methodology used in the validation study.

The eleven L-mode plasma discharges analyzed in this study are summarized in Table 1. All Alcator CMod discharges operated at $5.4 \mathrm{~T}$ magnetic field on axis and were heated with hydrogen minority ion cyclotron resonance heating. All ASDEX Upgrade discharges operated at $2.5 \mathrm{~T}$ on axis and were heated with electron cyclotron heating, with neutral beam blips for charge exchange measurements.. The first four Alcator CMod discharges had both $\widetilde{T} / T$ and $\chi_{e}^{\text {pert }}$ measurements at the same radius, while the fifth had only $\widetilde{T} / T$ measurements. All six ASDEX Upgrade discharges had $\widetilde{T} / T$ and $\chi_{e}^{\text {pert }}$ measurements at different radii (always $\widetilde{T} / T$ at the outer radius and $\chi_{e}^{\text {pert }}$ at the inner radius), so validation is performed at two radial locations for each of these discharges. Plasma current, heating power, and density was varied between the discharges, as is listed in Table 1 .

The large number of discharges and the goal of developing broader criteria for the importance of multi-scale effects dictate a methodology that differs from past validation studies. For each validation case, TGLF is run in both ion- and multi-scale configurations. The multi-scale TGLF configuration includes modes from $k_{\theta} \rho_{s}=0.1$ to 24.0 (the standard wavenumber spectrum in TGLF). The ion-scale TGLF configuration includes modes from $k_{\theta} \rho_{s}=0.08$ to 1.0. Note that both of these settings are run with the 'SAT1 ' turbulence saturation rule [7].

In addition to running TGLF in both ionand multi-scale settings for each discharge, this study will change which inputs are allowed to vary within experimental uncertainty, and which validation constraints are applied to each simulation. Traditionally, only the ion temperature gradient, or the ion and electron temperature gradients, are varied as inputs within experimental uncertainty in order to see if the simulations can match the electron and ion heat fluxes within experimental uncertainty. Generally,

\begin{tabular}{|c|c|c|c|c|c|}
\hline Machine & Discharge & $\begin{array}{c}I_{p} \\
(\mathrm{MA})\end{array}$ & $\begin{array}{l}P_{a u x} \\
(\mathrm{MW})\end{array}$ & Radius & $\begin{array}{c}n_{e} \\
\left(10^{19}\right. \\
\left./ \mathrm{m}^{3}\right)\end{array}$ \\
\hline \multirow{5}{*}{$\begin{array}{l}\text { Alcator } \\
\text { C-Mod }\end{array}$} & 1120706008 & 0.8 & 1.2 & 0.75 & 6.52 \\
\hline & 1120706017 & 0.8 & 1.2 & 0.75 & 8.83 \\
\hline & 1120706018 & 0.8 & 1.2 & 0.75 & 10.0 \\
\hline & 1120706019 & 0.8 & 1.2 & 0.75 & 6.28 \\
\hline & 1120706030 & 0.8 & 4.5 & 0.75 & 6.74 \\
\hline \multirow{12}{*}{$\begin{array}{c}\text { ASDEX } \\
\text { Upgrade }\end{array}$} & \multirow{2}{*}{33585} & \multirow{2}{*}{1.0} & \multirow{2}{*}{0.7} & 0.75 & 1.41 \\
\hline & & & & 0.49 & 1.99 \\
\hline & \multirow{2}{*}{34301} & \multirow{2}{*}{0.8} & \multirow{2}{*}{0.3} & 0.70 & 1.99 \\
\hline & & & & 0.39 & 2.46 \\
\hline & \multirow{2}{*}{34303} & \multirow{2}{*}{0.6} & \multirow{2}{*}{0.3} & 0.70 & 1.58 \\
\hline & & & & 0.30 & 2.70 \\
\hline & \multirow{2}{*}{34309} & \multirow{2}{*}{1.0} & \multirow{2}{*}{0.6} & 0.70 & 1.85 \\
\hline & & & & 0.50 & 2.47 \\
\hline & \multirow{2}{*}{34508} & \multirow{2}{*}{0.6} & \multirow{2}{*}{0.5} & 0.70 & 1.80 \\
\hline & & & & 0.30 & 2.91 \\
\hline & \multirow{2}{*}{34623} & \multirow{2}{*}{0.6} & \multirow{2}{*}{0.6} & 0.65 & 2.50 \\
\hline & & & & 0.27 & 3.90 \\
\hline
\end{tabular}

Table 1: Summary of plasma parameters for the five Alcator C-Mod and six ASDEX Upgrade discharges used in this study. All of the Alcator C-Mod discharges operated at $5.4 \mathrm{~T}$ magnetic field on axis, and all of the ASDEX Upgrade discharges at 2.5 T.

the gradients are input in the form of normalized gradient scale lengths, $a / L_{y}=-(a / y)(d y / d r)$, where $y$ is the parameter of interest and $a$ is the plasma minor radius. This study, however, will also vary the density gradient $\left(a / L_{n_{e}}\right)$ and the effective charge $Z_{\text {eff }}$ within experimental uncertainty in order to see if the code can match the validation constraints. These four parameters were chosen due to their independence. In particular, for each discharge and each scale setting (ion or multi) of TGLF, either only temperature gradients, or temperature gradients, density gradient, and effective charge were varied (either two inputs were varied or four inputs were varied). Input variations were all performed within the VITALS framework [44, which efficiently optimizes inputs in order to match constraints.

Finally, as described above, this work uses four validation constraints: electron heat flux, ion heat flux, electron temperature fluctuations, and electron perturbative thermal diffusivity.

Ion and electron heat fluxes are calculated as 
standard outputs from TGLF [6, 7]. The perturbative thermal diffusivity is calculated by artificially varying the input electron temperature gradient in TGLF up and down by $12 \%$, and then calculating the slope of the resulting change in the electron heat flux 18, 19. Finally, the electron temperature fluctuation level is calculated by integrating the output fluctuation spectrum up to the appropriate wavenumber for each case. For the ASDEX Upgrade cases, a correction is applied to this integrated value in order to account for differences between total and perpendicular temperature fluctuation levels [45]. This correction is necessary since CECE measures perpendicular temperature fluctuation levels, but TGLF outputs total temperature fluctuation levels. The higher collisionality of the Alcator C-Mod cases make this correction unnecessary.

All told, the variations in inputs and outputs are summarized as follows: ion- or multi-scale TGLF, two inputs $\left(a / L_{T_{e}}\right.$ and $\left.a / L_{T_{i}}\right)$ or four inputs $\left(a / L_{T_{e}}, a / L_{T_{i}}\right.$, $a / L_{n_{e}}$ and $\left.Z_{e f f}\right)$, and four different combinations of constraints $\left(Q_{e}\right.$ and $Q_{i} ; Q_{e}, Q_{i}$, and $\widetilde{T} / T ; Q_{e}, Q_{i}$, and $\chi_{e}^{\text {pert }}$; or $Q_{e}, Q_{i}, \widetilde{T} / T$ and $\left.\chi_{e}^{\text {pert }}\right)$. This totals 16 separate studies for each radius of each discharge (though some discharges or radii had only $\widetilde{T} / T$ or $\chi_{e}^{\text {pert }}$, but not both simultaneously).

In summary, the turbulent transport code TGLF, run through the VITALS framework, will be validated in 17 plasma conditions from 11 discharges on 2 machines (6 discharges have 2 locations). At each radial location, ion- and multi-scale TGLF will be validated, varying either two or four input parameters. Depending on the measurements available at each location, either two sets of constraints or four sets of constraints will be applied to both TGLF models and sets of inputs.

\section{Validation Results}

Consider frist the straightforward validation results of this study, concerning whether or not a given simulation was able to match a given set of experimental constraints within uncertainty. Table 2 shows the full validation results for each combination of scale, varied inputs, and applied constraints for each plasma condition. In this table, a green checkmark indicates agreement between the simulation and experiment within experimental uncertainty. A red $\mathrm{X}$ indicates disagreement. An empty grey box indicates that the particular set of experimental measurements for that column was not available at that radial location for that discharge. Inputs were only varied within experimental uncertainty.

While this table contains a somewhat overwhelming amount of information, a few trends are highlighted before going into more detailed analysis. First, as one would expect, for a given model and discharge, adding more constraints reduces the chance of agreement, while varying more inputs increases the chance for agreement.

One significant result of this validation study is that, when one allows all four inputs to vary within uncertainty, the multi-scale TGLF simulations matched all available validation constraints for every plasma condition. This can be seen by looking at the furthest right colored cell in each row (the multi-scale model varying four inputs and with as many constraints as are available). In this sense, this study has successfully validated TGLF on 11 plasma discharges on 2 machines, using four validation constraints. This is discussed further in Section 5 .

On the other hand, the ion-scale model agreed with all available constraints in some cases, but not others, as seen in the right-most filled cells in the ion-scale column for each row. The question of when multi-scale effects are important for turbulent transport simulations is exactly the question of why some ion-scale simulations can match the validation constraints while others cannot. This question is addressed quantitatively later in this section using two criteria developed as part of this work.

Finally, note that in nearly all cases, the multiscale model performs better than the ion-scale model. This is perhaps not surprising, as it contains more physics and one would expect it to perform better. There are a few exceptions to this, particularly when only temperature gradients are varied and only heat fluxes are matched, but it is likely that these represent exactly the fortuitous agreement that one uses additional validation constraints in order to avoid.

\subsection{Validation Metric and Criteria for Importance of Multi-Scale Effects}

In order to analyze the question of when multiscale effects are important in turbulent transport simulations, a quantitative validation metric is used to evaluate the performance of the ion- and multi-scale simulations.

This study uses a modified version of the Ricci validation metric, $\chi_{\text {Ricci }}$ [46, to quantify the differences between the ion- and multi-scale models 7 For each case, $\chi_{\text {Ricci }}$ is essentially a weighted sum of the differences between the simulated and experimental constraint values, normalized by the uncertainty of the experimental measurements. No one constraint dominated the total $\chi_{\text {Ricci }}$ in the cases considered here. This Ricci metric is bound between 0 (perfect

$\ddagger$ To address a possible confusion, note that $\chi_{\text {Ricci }}$ is not a diffusivity (unlike $\chi_{e}^{\text {pert }}$ ) and is unitless. The symbol $\chi$ is only used to connect with past literature [46]. 


\begin{tabular}{|c|c|c|c|c|c|c|c|c|c|c|c|c|c|c|c|c|c|c|}
\hline \multirow{8}{*}{ 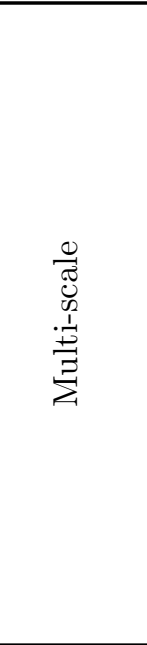 } & \multirow{4}{*}{ 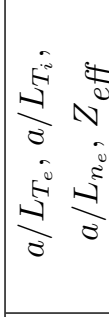 } & 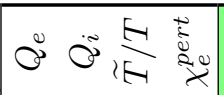 & $>$ & $>$ & $>$ & $>$ & & & & & & & & & & & & \\
\hline & & $\dot{\sigma} \tilde{\sigma}$ & $>$ & $>$ & $>$ & $>$ & & & $>$ & & $>$ & & $>$ & & $>$ & $>$ & & $>$ \\
\hline & & $\ddot{\sigma} \tilde{\sigma} \frac{E}{1 E}$ & $>$ & $>$ & $>$ & $>$ & $>$ & $>$ & & $>$ & & $>$ & & $>$ & & $>$ & $>$ & \\
\hline & & $\ddot{\sigma}$ & $>$ & $>$ & $>$ & $>$ & $>$ & $>$ & $>$ & $>$ & $>$ & $>$ & $>$ & $>$ & $>$ & $>$ & $>$ & $>$ \\
\hline & \multirow{4}{*}{$\begin{array}{l}\frac{k}{8} \\
0 \\
\frac{1}{3} \\
\frac{0}{3}\end{array}$} & 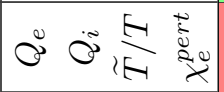 & $x$ & $x$ & $>$ & $>$ & & & & & & & & & & & & \\
\hline & & $\ddot{\sigma} \ddot{\sigma}$ & $x$ & $>$ & $>$ & $>$ & & & $>$ & & $>$ & & $>$ & & $>$ & $>$ & & $x$ \\
\hline & & $\tilde{\sigma} \dot{\sigma} \frac{E}{1 E-1}$ & $x_{1}$ & $x$ & $>$ & $>$ & $x$ & $x$ & & $x$ & & $x$ & & $x$ & & $>$ & $>$ & \\
\hline & & $\ddot{\sigma}$ & $x$ & $>$ & $>$ & $>$ & $x$ & $>$ & $>$ & $>$ & $>$ & $>$ & $>$ & $>$ & $>$ & $>>$ & $>$ & $>$ \\
\hline \multirow{8}{*}{$\begin{array}{l}\stackrel{0}{\widetilde{J}} \\
\text { D } \\
0 \\
0 \\
0\end{array}$} & \multirow{4}{*}{ 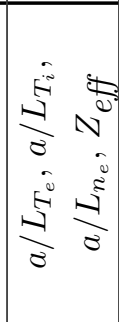 } & Oें & $x$ & $x$ & $x$ & $x_{1}$ & & & & & & & & & & & & \\
\hline & & $\ddot{\sigma} \dot{\sigma} \ddot{\tilde{\Xi}}$ & $>$ & $>$ & $>$ & $>$ & & & $>$ & & $x$ & & $x$ & & $x$ & $x$ & & $x$ \\
\hline & & $\dot{\sigma} \tilde{\sigma} \frac{E}{k-1}$ & $>$ & $x$ & $>$ & $>$ & $>$ & $>$ & & $>$ & & $>$ & & $>$ & & $x$ & $x$ & \\
\hline & & ஸ் ஸ் & $>$ & $>$ & $>$ & $>$ & $>$ & $>$ & $>$ & $>$ & $>$ & $>$ & $>$ & $>$ & $>$ & $x_{1}>$ & $x$ & $x$ \\
\hline & & 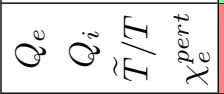 & $x$ & $x$ & $x$ & $x$ & & & & & & & & & & & & \\
\hline & $\frac{1}{8}$ & 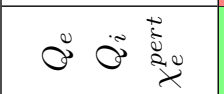 & $>$ & $>$ & $>$ & $>$ & & & $>$ & & $x$ & & $x$ & & $x$ & $x$ & & $x$ \\
\hline & $\frac{8}{\sqrt[3]{3}}$ & $\dot{\sigma} \dot{\sigma} \frac{E}{1 E-1}$ & $x$ & $x$ & $x$ & $>$ & $x$ & $>$ & & $>$ & & $x$ & & $x$ & & $x$ & $x$ & \\
\hline & & $\ddot{\sigma}$ & $>$ & $>$ & $>$ & $>$ & $x$ & $>$ & $>$ & $>$ & $x$ & $>$ & $x$ & $>$ & $>$ & $x_{1} \times$ & $x$ & $x$ \\
\hline 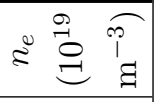 & & & 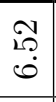 & $\begin{array}{l}\mathscr{0} \\
\infty \\
\infty\end{array}$ & $\stackrel{0}{\circ}$ & \begin{tabular}{l}
$\infty$ \\
\multirow{N}{*}{} \\
0
\end{tabular} & $\stackrel{+}{5}$ & $\underset{\rightleftarrows}{\stackrel{F}{\longrightarrow}}$ & $\stackrel{\mathscr{P}}{\mathscr{\leftrightarrow}}$ & 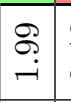 & $\begin{array}{l}\stackrel{\oplus}{H} \\
i \\
\end{array}$ & 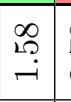 & $\begin{array}{l}R \\
i \\
i\end{array}$ & $\begin{array}{l}20 \\
\infty \\
- \\
\end{array}$ & $\begin{array}{l}\text { ने } \\
\text { ¿ } \\
\text { i }\end{array}$ & 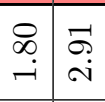 & \begin{tabular}{l|l}
$i$ \\
$i$ \\
$i$ \\
$i$
\end{tabular} & $\begin{array}{l}8 \\
8 \\
\infty\end{array}$ \\
\hline$\frac{2}{\grave{2}}$ & & & \begin{tabular}{|l|}
29 \\
0 \\
0
\end{tabular} & $\stackrel{10}{\stackrel{10}{0}}$ & \begin{tabular}{|l}
12 \\
10 \\
0
\end{tabular} & $\begin{array}{ll}12 \\
12 \\
0\end{array}$ & \begin{tabular}{|l|}
20 \\
5 \\
0
\end{tabular} & 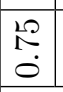 & $\stackrel{\mathscr{P}}{+}$ & \begin{tabular}{|l|l|}
$R$ & \\
0 & \\
\end{tabular} & 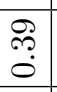 & $\begin{array}{ll} \\
0 \\
0\end{array}$ & 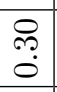 & $\begin{array}{l}R \\
0 \\
0\end{array}$ & 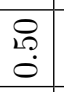 & \begin{tabular}{l|l}
$\stackrel{0}{0}$ & 0 \\
0 & 3 \\
0
\end{tabular} & \begin{tabular}{|l|}
12 \\
0 \\
0 \\
0
\end{tabular} & 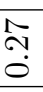 \\
\hline 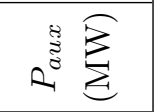 & & & $\stackrel{\sim}{-}$ & 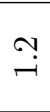 & $\stackrel{\sim}{ }$ & 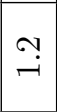 & $\stackrel{L}{L}$ & $\hat{0}$ & & $\stackrel{?}{0}$ & & $\stackrel{?}{0}$ & & $\stackrel{0}{0}$ & & $\stackrel{10}{0}$ & $\stackrel{0}{0}$ & \\
\hline 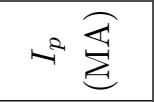 & & & $\stackrel{\infty}{0}$ & $\stackrel{\infty}{\circ}$ & $\stackrel{\infty}{\circ}$ & $\stackrel{\infty}{0}$ & $\stackrel{\infty}{\circ}$ & $\stackrel{0}{-}$ & & $\stackrel{\infty}{\circ}$ & & $\stackrel{\leftrightarrow}{\circ}$ & & $\stackrel{\circ}{-}$ & & $\stackrel{\varphi}{0}$ & $\stackrel{0}{0}$ & \\
\hline 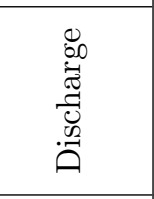 & & & 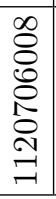 & 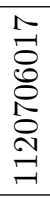 & 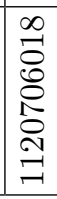 & 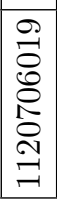 & 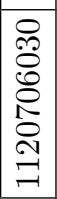 & $\begin{array}{l}10 \\
\infty \\
10 \\
i \\
i\end{array}$ & & 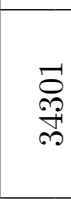 & & $\underset{\stackrel{m}{O}}{\stackrel{\infty}{F}}$ & & $\begin{array}{l}\stackrel{8}{\mathscr{P}} \\
\stackrel{\leftrightarrow}{*}\end{array}$ & & 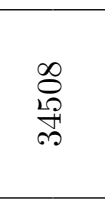 & 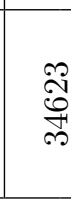 & \\
\hline 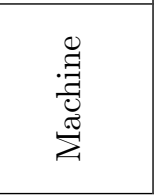 & $\begin{array}{l}\stackrel{0}{0} \\
\stackrel{\Xi}{\Xi}\end{array}$ & 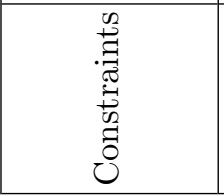 & & & & & & & & & & & 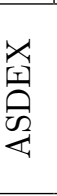 & 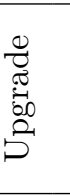 & & & & \\
\hline
\end{tabular}

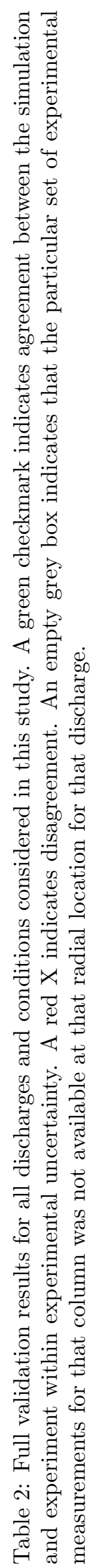


agreement) and 1 (disagreement). In order to analyze the difference between an ion- and multi-scale case, one takes the difference between the $\chi_{\text {Ricci }}$ values:

$\Delta \chi_{\text {Ricci }}=\chi_{i o n}-\chi_{m u l t i}$

where $\chi_{i o n}$ is the Ricci metric calculated for the ion-scale run and $\chi_{\text {multi }}$ is calculated for the multi-scale run of a plasma condition.

With this definition, a value of $\Delta \chi_{\text {Ricci }}=0$ indicates that the ion- and multi-scale models match experimental constraints equally well (though they may do so using different input parameters). A value of 1 indicates that the multi-scale model performs better than the ion-scale model (multi-scale effects are important). It is also possible to have negative values of $\Delta \chi_{\text {Ricci }}$, indicating that the ion-scale model performs better, though, outside of small variations in optimization, the multi-scale model should in theory perform better in all cases. This particular study chooses $\Delta \chi_{\text {Ricci }}=0.1$ as the cutoff between multi-scale effects being important or unimportant.

In the analysis that follows, $\Delta \chi_{\text {Ricci }}$ will only be calculated for the simulation cases where all available validation constraints have been applied and all four inputs have been allowed to vary within uncertainty (the furthest right colored set of boxes in Table 2). Each plasma discharge and position will therefore have a single value of $\Delta \chi_{\text {Ricci }}$.

This quantification was used to develop criteria with which one is able to determine whether or not multi-scale effects will be important in a plasma discharge, without performing nonlinear simulations. These criteria are based on properties of the linear growth rates of the plasmas, similar to some past work, which suggested that $\gamma_{\text {high-k }} / \gamma_{\text {low-k }}$ might be one such criterion [13, 16, 19. In this criterion, $\gamma_{h i g h-k}$ is the peak linear growth rate in the electron scales $\left(k_{y} \rho_{s} \gtrsim\right.$ $2.0)$, and $\gamma_{\text {low- } k}$ is the peak linear growth rate in the ion scales $\left(k_{y} \rho_{s} \lesssim 2.0\right)$. Other work suggested that one should instead use $\left(\operatorname{MAX}(\gamma / k)_{\text {high }}\right) /\left(\operatorname{MAX}(\gamma / k)_{l o w}\right)$, for which one takes the peak of the linear growth rate spectrum normalized by the wavenumber above $k_{y} \rho_{s}>1.0$, divided by the peak at low wavenumber 8. Those studies, however, were based on a limited set of discharges, and so it was difficult to draw any broad conclusions.

The work presented here shows plasmas must simultaneously satisfy two criteria for ion-scale simulations to be sufficient.

The first of these two criteria is similar to that proposed in Reference [8, defined here as:

$\Gamma_{\text {high } / \text { low }}=\left(\gamma_{\text {high-k}} / k_{\text {high }}\right) /\left(\gamma_{\text {low }-k} / k_{\text {low }}\right)$

where $k_{h i g h}$ is the wavenumber of the peak high-k linear growth rate, and $k_{\text {low }}$ is the wavenumber of the

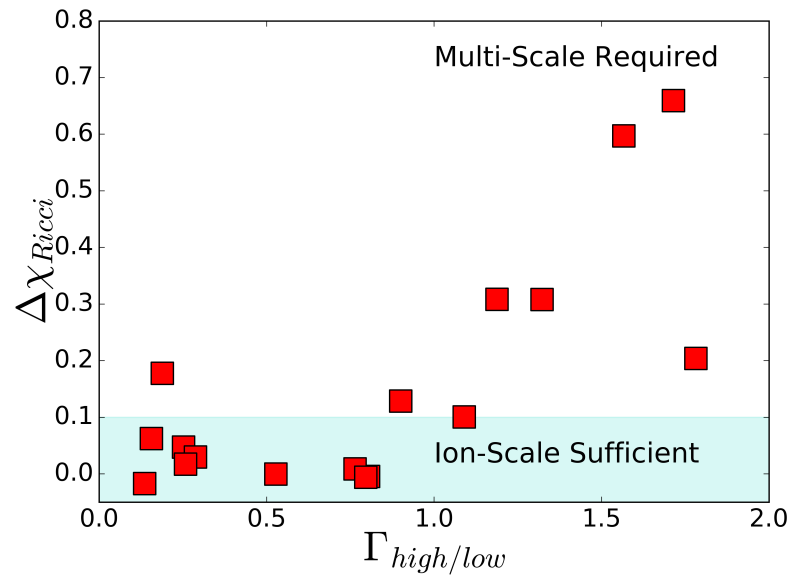

Figure 1: The importance of multi-scale effects, represented by $\Delta \chi_{\text {Ricci }}$, plotted against the ratio of the wavenumber-normalized peak growth rate ratio, given by $\Gamma_{\text {high } / \text { low }}$. Each square is one validation case. The blue highlighted region, below $\Delta \chi_{\text {Ricci }}=0.1$, indicates that ion-scale simulations are sufficient.

peak low-k linear growth rate. For instances where there was no clear peak at low wavenumber (for a monotonically increasing linear growth spectrum), the linear growth rate at $k_{y} \rho_{s}=2$ was used as $\gamma_{\text {low- } k}$.

Figure 1 shows the results of plotting $\Delta \chi_{\text {Ricci }}$ against $\Gamma_{\text {high/low }}$, where each point represents one of the 17 plasma conditions described above. With a few exceptions (which are addressed by the second criterion), cases for which $\Gamma_{\text {high/low }}<1.0$ have $\Delta \chi_{\text {Ricci }}<0.1$ and the ion-scale TGLF model is sufficient. On the other hand, as $\Gamma_{\text {high/low }}$ increases above 1.0, $\Delta \chi_{\text {Ricci }}$ steadily increases, indicating that multi-scale effects become increasingly important.

The form of this criterion reveals which physical phenomena are dominantly responsible for the importance of multi-scale effects. It is generally accepted that larger linear growth rates at electron scales lead to more significant electron-scale contributions to the overall heat flux and turbulence characteristics. In addition to contributions from purely electron-scale phenomena, multi-scale simulations also include crossscale coupling between the ion- and electron-scales, moderated by zonal flows $[7,8,10,11$.

A normalization of $1 / k$ indicates that zonal flow mixing and its role in coupling ion- and electronscales is the most important physical mechanism in determining when multi-scale effects are important 8. In contrast, a $1 / k^{2}$ normalization would have indicated that the mixing length of a given turbulent mode was dominantly important 7]. The form of this criterion therefore reveals that coupling between ion- and electron-scales, instead of the inclusion of purely electron-scale transport, is most relevant to 


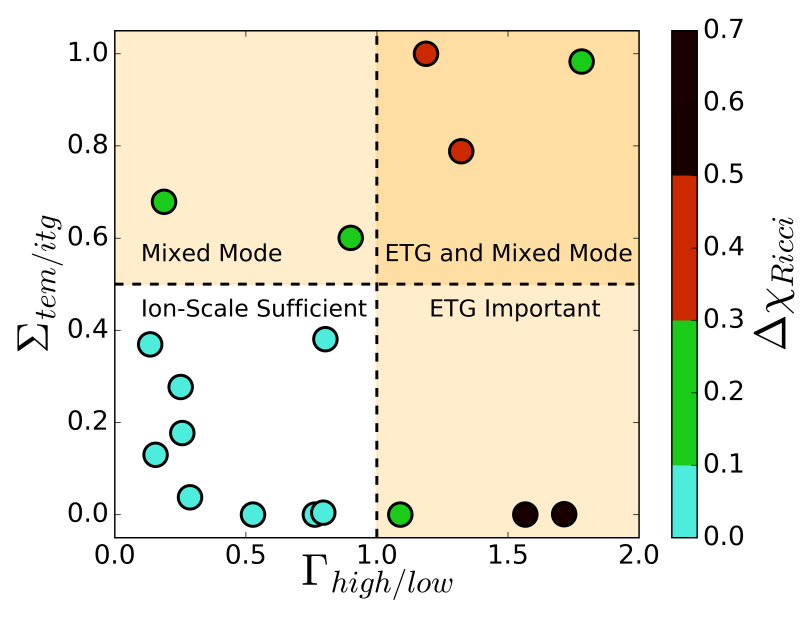

Figure 2: Criteria for the importance of multi-scale effects, $\Gamma_{\text {high } / \text { low }}$ and $\Sigma_{\text {tem } / \text { itg }}$, with color scale showing $\Delta \chi_{\text {Ricci }}$. Values below 0.1 , indicating that ion-scale simulations are sufficient, are turquoise. The shaded regions indicate that multi-scale effects are important due to the breaking of one or both criteria.

the importance of multi-scale simulations. This is consistent with past work revealing that linearly adding ion- and electron-scale simulations is not equivalent to running a multi-scale simulation.

The second criterion is based on the relative importance of ITG (ion temperature gradient) and TEM (trapped electron) modes at ion-scales. This criterion is defined as:

$\Sigma_{t e m / i t g}=\exp \left(\frac{-\left(\frac{\gamma_{T E M} / k_{T E M}}{\gamma_{I T G} / k_{I T G}}-1.0\right)^{2}}{0.09}\right)$

where $\gamma_{T E M}$ is the peak linear growth rate of the TEM at low-k, $k_{T E M}$ is the wavenumber of this peak, $\gamma_{I T G}$ is the peak linear growth rate of the ITG, and $k_{I T G}$ is the wavenumber of the peak. This measures how close the peak ITG and TEM growth rates (normalized by the wavenumber) are to one another, with 1 indicating that they are very close, and 0 indicating that one dominates. At the cost of mathematical simplicity, the metric was constructed such that it varies from zero to one, and the 0.09 in the denominator was chosen such that the cutoff of $\Delta \chi_{\text {Ricci }}=0.1$ occurs at $\Sigma_{\text {tem } / \text { itg }}=0.5$.

Figure 2 shows each plasma condition on a twodimensional plot of $\Gamma_{\text {high/low }}$ and $\Sigma_{\text {tem/itg }}$, with the value of $\Delta \chi_{\text {Ricci }}$ represented by color. Turquoise represents cases where ion-scale simulations are sufficient $\left(\Delta \chi_{\text {Ricci }}<0.1\right)$, and green, red, and black represent increasing importance of multi-scale effects. This figure reveals that ion-scale simulations are sufficient for all plasmas in the lower left quadrant $\left(\Gamma_{\text {high/low }}<1.0\right.$ and $\left.\Sigma_{\text {tem } / \text { itg }}<0.5\right)$. If either criterion is broken, however, multi-scale effects become important.

The second criterion's interpretation also concerns the importance of cross-scale coupling in determining the final turbulent state of the plasma. Specifically, even in cases with less strongly driven electron-scale turbulence (that don't break the first criterion), if the ion-scale turbulence has no clearly dominant mode (ITG and TEM compete for dominance), cross-scale coupling is still important and multi-scale simulations are necessary. In other words, when there is no clearly dominant mode at ion-scales, a small amount of crossscale coupling can determine which mode becomes dominant.

Taken together, these criteria mean that if either electron-scale turbulence is sufficiently driven, or ionscale turbulence is particularly susceptible to crossscale coupling contributions, then multi-scale effects are important, and ion-scale simulations do not adequately model the plasma.

\subsection{Profile Prediction}

As an example of the practical implications of these criteria, consider using TGLF to predict temperature profiles. This is done iteratively through the TGYRO framework [4], adjusting local temperature gradients until the simulated heat flux matches the experimental heat flux, and then integrating to get the full profile. Unlike in the validation above, effective charge and density gradients were not changed. Profiles are predicted using both the ion- and multi-scale configurations of TGLF for two plasmas: one in which the criteria just derived suggest that ion-scale simulations should be sufficient, and one in which these criteria suggest that multi-scale simulations are necessary. These two plasmas are ASDEX Upgrade discharges 33585 and 34623, both of which were included in the validation study and are described in greater detail above.

Each of these profile predictions was performed with TGLF simulations at 8 radial points, ranging from $\rho_{\text {tor }}=0.2$ or 0.3 to $\rho_{\text {tor }}=0.8$, depending on how large the sawtooth mixing radius was. Inside of $\rho_{\text {tor }}=0.2$ or 0.3 transport is heavily influenced by the sawtooth oscillation, which is not included in the TGLF simulations as they are implemented here. Outside of $\rho_{\text {tor }}=0.8$ edge effects become important, and transport cannot be treated with traditional core turbulence modeling without considerable care. The predicted profiles are therefore pinned at the experimentally measured profile at $\rho_{\text {tor }}=0.8$.

Figure 3 shows the results of profile predictions for two of the ASDEX Upgrade discharges considered in the validation study above, 33585 and 34623. Discharge 33585 had $\Gamma_{\text {high } / \text { low }}=0.18$ and 


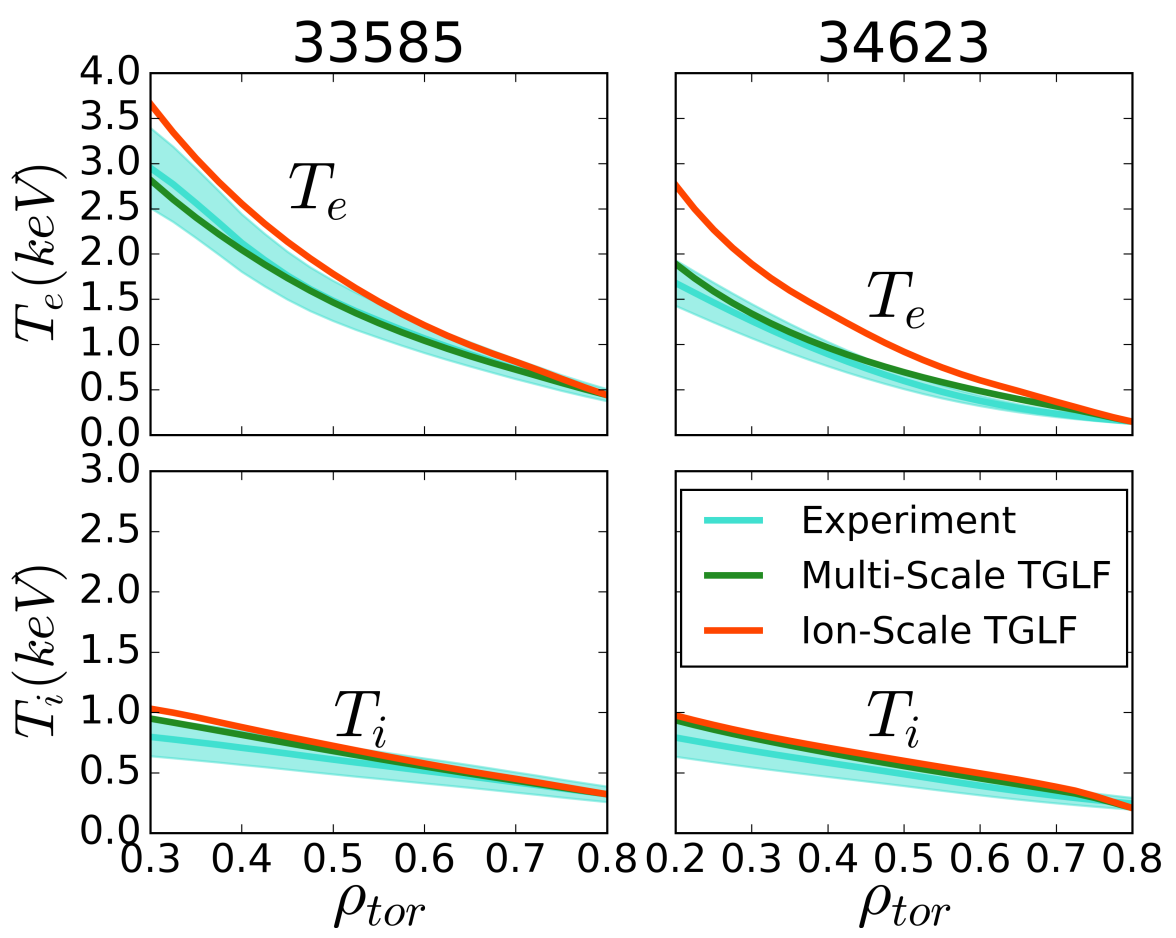

Figure 3: Temperature profile predictions for two ASDEX Upgrade discharges. Top row is discharge 33585, bottom is 34623. Left column in electron temperature, right is ion temperature. Experimental profiles are shown in turquoise, with uncertainty represented by the shaded region. Multi-scale TGLF predictions are shown in green and ion-scale in red. The inner sawtooth region and outer edge region are shaded out.

$\Sigma_{\text {tem } / \text { itg }}=0.26$ at $\rho_{\text {tor }}=0.50$. This discharge satisfies both criteria. Discharge 34623 had $\Gamma_{\text {high } / \text { low }}=1.7$ and $\Sigma_{\text {tem } / \text { itg }}=0.001$ at $\rho_{\text {tor }}=0.27$. This discharge violates the first criterion.

As this figure shows, multi-scale TGLF was able to predict the ion and electron temperature profiles to within experimental uncertainty for both discharges, with only a small deviation from the experimental electron temperature of discharge 34623 around $\rho_{\text {tor }} \approx$ 0.7. Allowing the density gradient or effective charge to vary would likely resolve this discrepancy. On the other hand, ion-scale TGLF predictions agreed fairly well with experimental measurements in 33585, only slightly outside of uncertainty, but were significantly higher than the experimental electron temperature in discharge 34623 . These predictions were robust to the spatial resolution of the TGYRO run and to the edge pinning location (moved in or out by $0.05 \rho_{\text {tor }}$ ).

The results of these predictions are consistent with what one would expect based on the two criteria developed in this paper. Discharge 33585 satisfies both criteria, indicating the ion-scale simulations should be sufficient, and the ion-scale prediction is accordingly fairly close to the experimental measurement. On the other hand, discharge 34623 violates the first criteria with $\Gamma_{\text {high } / \text { low }}=1.7>1.0$, indicating that multi-scale effects are important and that one should not trust the result of ion-scale simulations. This is consistent with the poor performance of the ion-scale model.

\section{Implications and Discussion}

Turbulent transport models have improved enormously over the last decade or so, and are now in some cases able to accurately model experimental behavior. The validation effort for these models is, however, far from complete, and only after these models have been exhaustively validation on current machines should one trust them to predict the performance of future machines. The work presented here described a new type of validation study, validating a turbulent transport model on many discharges on multiple machines using multiple validation constraints. In some sense this is a breadth approach, rather than depth into a single discharge. In order to get a better sense of where current turbulent transport models fare well, and where they fail to capture experimental results, it is likely that future validation studies will also follow the pattern of looking at many discharges and many machines. This approach will give a clearer sense of whether or not current models can be trusted to predict future machines. 
One of the concrete results of this study is that multi-scale TGLF matched all of the validation constraints applied in this study within experimental uncertainty on all discharges on both machines. This is clear evidence of the strength of the TGLF model, but there is of course much more work to be done. The discharges analyzed here were all relatively standard, electron-heated L-mode plasmas. To validate TGLF more broadly, one must work with additional discharges that cover a much larger parameter space, including H-modes, I-modes, high $\beta$ plasmas, ion-heated plasmas, etc. In addition, while this study included two machines, such validation efforts must also expand to other machines, in order to take advantage of the variety of experimental facilities around the world.

Another product of the work presented here is a set of criteria to determine when multi-scale effects are important in turbulent transport. From a practical perspective, one may ask why it isn't always better to just run a multi-scale simulation, and to forget ion-scale simulations all together. In the case of TGLF, this is a valid point. Running multi-scale TGLF takes only marginally longer than running ionscale TGLF, and the results are clearly superior. If one wants to predict the performance of a future machine that is far away in parameter space from current machines, however, it may be that a full nonlinear gyrokinetic simulation is far more reliable than a quasi-linear model such as TGLF that requires a tuned nonlinear saturation rule. For this reason, it is likely that the most trusted simulations of future machines will involve nonlinear gyrokinetic models, at least for the time being, and not a quasi-linear model. For gyrokinetic codes, the cost of running a multi-scale simulation is significant, and can in some cases be prohibitive. The criteria developed in this work allow one to determine whether or not multi-scale gyrokinetic simulations are necessary, before actually running them. Such knowledge ensures that one doesn't unnecessarily run multi-scale simulations if they are not required, while remaining confident in the results of ion-scale simulations in those cases.

\section{Conclusions}

This work presented a validation study of the TGLF turbulent transport model on 11 plasma discharges from Alcator C-Mod and ASDEX Upgrade, and used these results to develop criteria to differentiate cases where multi-scale effects are important and where ion-scale simulations are sufficient. The validation study showed that multi-scale TGLF agreed with all available constraints within uncertainty for all of the experimental conditions, after varying the input electron temperature gradient, ion temperature gradient, density gradient, and effective charge within uncertainty. Future work will include additional discharges that cover a much larger parameter space, including $\mathrm{H}$-modes, I-modes, additional tokamaks, etc. The results led to a set of two criteria with which one can determine the importance of multi-scale effects, one based on the ratio of normalized high-k and low$\mathrm{k}$ growth rates, and the other based on the ratio of normalized ITG and TEM growth rates at lowk. Example profile predictions were made for two discharges, one which violates the first criterion and one which satisfies, illustrating the importance of using the correct model when the criteria are violated. This work has provided further insight into the fundamental nature of multi-scale turbulent interactions and will guide model selection for predictions of future fusion devices.

\section{Acknowledgements}

The authors would like to thank Gary Staebler for insightful discussions about TGLF and this work. This work has been carried out within the framework of the EUROfusion Consortium and has received funding from the Euratom research and training programme 2014-2018 and 2019-2020 under grant agreement No 633053. The views and opinions expressed herein do not necessarily reflect those of the European Commission. This work is also supported by the US DOE under Grants DE-SC0006419, DE-FC0299ER54512-CMOD, and DE-SC0017381. In addition, this research is supported by the US DoD and the Air Force Office of Scientific Research under the National Defense Science and Engineering Graduate (NDSEG) Fellowship, 32 CFR 168a.

\section{References}

[1] AIAA 1998 Guide for the verification and validation of computational fluid dynamics simulations Tech. Rep. AIAA G-077-1998 (2002) The American Institute of Aeronautics and Astronautics

[2] Greenwald M 2010 Phys. Plasmas 17058101

[3] Holland C 2016 Phys. Plasmas 23060901

[4] Jenko F, Dorland W and Kotschenreuther M 2000 Phys. Plasmas 71904

[5] Candy J and Waltz R E 2003 J. Comput. Phys. 186 545581

[6] Staebler G M, Kinsey J E and Waltz R E 2007 Phys. Plasmas 14055909

[7] Staebler G M, Candy J, Howard N T and Holland C 2016 Phys. Plasmas 23062518

[8] Staebler G M, Howard N T, Candy J and Holland C 2017 Nucl. Fusion $\mathbf{5 7} 066046$

[9] Howard N T, White A E, Reinke M L, Greenwald M, Holland C, Candy J and Walk J 2013 Nucl. Fusion 53 123011

[10] Howard N T, Holland C, White A E, Greenwald M and Candy J 2014 Phys. Plasmas 21112510 
[11] Maeyama S, Idomura Y, Watanabe T H, Nakata M, Yagi M, Miyato N, Ishizawa A and Nunami M 2015 Phys. Rev. Lett. 114255002

[12] Howard N T, Holland C, White A E, Greenwald M and Candy J 2016 Nucl. Fusion 56014004

[13] Howard N T, Holland C, White A E, Greenwald M, Candy J and Creely A J 2016 Phys. Plasmas 23056109

[14] Holland C, Howard N and Grierson B 2017 Nucl. Fusion 57066043

[15] Maeyama S, Watanabe T H and Ishizawa A 2017 Phys. Rev. Lett. 119195002

[16] Howard N T, Holland C, White A E, Greenwald M, Rodriguez-Fernandez P, Candy J and Creely A J 2018 Plasma Phys. Controlled Fusion 60014034

[17] Creely A J, Howard N T, Rodriguez-Fernandez P, NCao, Hubbard A E, Hughes J W, Rice J E, White A E, Candy J, Staebler G M, Conway G D, Freethy S J and Sung C 2017 Phys. Plasmas 24056104

[18] Creely A J, White A E, Edlund E M, Howard N T and Hubbard A E 2016 Nucl. Fusion 56036003

[19] Creely A J, Görler T, Conway G D, Freethy S J, Howard N T, Schneider P A, White A E, Willensdorfer M and the ASDEX Upgrade Team 2018 Nucl. Fusion 58126001

[20] Greenwald M, Bader A, Baek S, Bakhtiari M, Barnard H, Beck W, Bergerson W, Bespamyatnov I, Bonoli P, Brower D, Brunner D, Burke W, Candy J, Churchill M, Cziegler I, Diallo A, Dominguez A, Duval B, Edlund E, Ennever P, Ernst D, Faust I, Fiore C, Fredian T, Garcia O, Gao C, Goetz J, Golfinopoulos T, Granetz R, Grulke O, Hartwig Z, Horne S, Howard N, Hubbard A E, Hughes J, Hutchinson I, Irby J, Izzo V, Kessel C, LaBombard B, Lau C, Li C, Lin Y, Lipschultz B, Loarte A, Marmar E, Mazurenko A, McCracken G, McDermott R, Meneghini O, Mikkelsen D, Mossessian D, Mumgaard R, Myra J, Nelson-Melby E, Ochoukov R, Olynyk G, Parker R, Pitcher S, Podpaly Y, Porkolab M, Reinke M, Rice J, Rowan W, Schmidt A, Scott S, Shiraiwa S, Sierchio J, Smick N, Snipes J A, Snyder P, Sorbom B, Stillerman J, Sung C, Takase Y, Tang V, Terry J, Terry D, Theiler C, Tronchin-James A, Tsujii N, Vieira R, Walk J, Wallace G, White A E, Whyte D G, Wilson J, Wolfe S, Wright G, Wright J, Wukitch S and Zweben S 2014 Phys. Plasmas 21110501

[21] Kallenbach A, the ASDEX Upgrade Team and the EUROfusion MST1 Team 2017 Nucl. Fusion 57102015

[22] O'Shea J, Hubbard A E and Group A C M 1995 9th Joint Workshop on ECE and ECRH, Borrego Springs p 7

[23] O'Shea P J L 1997 Ph.D. thesis Massachusetts Institute of Technology

[24] Hughes J W, Mossessian D, Zhurovich K, DeMaria M, Jensen K and Hubbard A 2003 Rev. Sci. Instrum. $\mathbf{7 4}$ 1667

[25] Ince-Cushman A, Rice J E, Bitter M, Reinke M L, Hill K W, Gu M F, Eikenberry E, Broennimann C, Scott S, Podpaly Y, Lee S G and Marmar E S 2008 Rev. Sci. Instrum. 79 10E302

[26] Foord M E, Marmar E S and Terry J L 1982 Rev. Sci. Instrum. 53 (9) 1407

[27] Lao L, John H S, Stambaugh R D, Kellman A G and Pfeiffer W 1985 Nucl. Fusion 251611

[28] Willensdorfer M, Denk S S, Strumberger E, Suttrop W, Vanovac B, Brida D, Cavedon M, Classen I, Dunne M, Fietz S, Fischer R, Kirk A, Laggner F M, Liu Y Q, Odstrcil T, Ryan D A, Viezzer E, Zohm H, Luhmann I C, the ASDEX Upgrade Team and Team T E M 2016 Plasma Phys. Control. Fusion 58114004

[29] Murmann H, Goetsch S, Roehr H, Salzmann H and Steuer K H 1992 Rev. Sci. Instrum. 634941

[30] Kurzan B and Murmann H D 2011 Rev. Sci. Instrum. 82 103501
[31] Viezzer E, Puetterich T, Dux R, McDermott R M and the ASDEX Upgrade Team 2012 Rev. Sci. Instrum. 83 103501

[32] Meister H, Fischer R, Horton L D, Maggi C F, Nishijima D, the ASDEX Upgrade Team, Giroud C, Zastrow K, Contributors J E and Zaniol B 2004 Rev. Sci. Instrum. 754097

[33] McCarthy P J 1999 Phys. Plasmas 63554

[34] See http://w3.pppl.gov/transp for full documentation concerning the TRANSP code.

[35] Goetz J, Lipschultz B, Graf M, Kurz C, Nachtrieb R, Snipes J and Terry J 1995 J. Nucl. Mater. 220971

[36] Mast K F, Vallet J C, Andelfinger C, Betzler P, Kraus H and Schramm G 1991 Rev. Sci. Instrum. 62744

[37] Sung C, White A E, Irby J H, Leccacorvi R, Vieira R, Oi C Y, Peebles W A and Nguyen X 2012 Rev. Sci. Instrum. 83 10E311

[38] Howard N T, Sung C and White A E 2014 Rev. Sci. Instrum. $8511 \mathrm{D} 811$

[39] Freethy S J, Conway G D, Classen I, Creely A J, Happel T, Khn A, Vanovac B and White A E 2016 Rev. Sci. Instrum. $8711 \mathrm{E} 102$

[40] Creely A J, Freethy S, Burke W M, Conway G D, Leccacorvi R, Parkin W C, Terry D R and White A E 2018 Rev. Sci. Instrum. 89053503

[41] Cardozo N J L 1995 Plasma Phys. Control. Fusion 37799

[42] Tubbing B J D, Cardozo N L and der Wiel M V 1987 Nucl. Fusion 271843

[43] Fredrickson E D, Austin M E, Groebner R, Manickam J, Rice B, Schmidt G and Snider R 2000 Phys. Plasmas 7 5051

[44] Rodriguez-Fernandez P, White A E, Creely A J, Greenwald M J, Howard N T, Sciortino F and Wright J C 2018 Fusion Science and Technology $\mathbf{7 4} 65$

[45] Freethy S J, Goerler T, Creely A J, Conway G D, Happel $\mathrm{T}$, Koenen C, Hennequin P, White A E and the ASDEX Upgrade Team 2018 Phys. Plasmas 25055903

[46] Ricci P, Theiler C, Fasoli A, Furno I, Gustafson K, Iraji D and Loizu J 2011 Phys. Plasmas 18032109

[47] Candy J, Holland C, Waltz R E, Fahey M R and Belli E 2009 Phys. Plasmas 16060704 